\title{
FACTORS AFFECTING THE FREQUENCY OF EXACERBATIONS IN ADULT PATIENTS WITH BRONCHIECTASIS
}

DOI: 10.36740/WLek202008124

\author{
Kateryna Gashynova, Kseniia Suska, Valeriia Dmytrychenko \\ OCCUPATIONAL DISEASES AND CLINICAL IMMUNOLOGY, SI "DNIPROPETROVSK MEDICAL ACADEMY OF THE HEALTH MINISTRY OF UKRAINE”, DNIPRO, UKRAINE
}

\begin{abstract}
The aim: This study aims to determine specific predictive factors of bronchiectasis exacerbations.

Materials and methods: 47 patients with bronchiectasis were prospectively recruited into the study in Dnipro, Ukraine. Following the number of exacerbations during the previous year, they were divided into two groups: frequent exacerbators - $\geq 3$ cases per year $(n=24)$ and non-frequent exacerbators $-<3$ cases per year $(n=23)$. Demographic and anthropometric data, medical history, smoking status, shortness of breath by Modified Medical Research Council Dyspnea Scale, sputum culture, respiratory function by computed spirometry, disease severity by Bronchiectasis Severity index and FACED scales were evaluated in both groups.

Results: The factors found to be independently associated with frequent exacerbations were: overweight, airway obstruction, longer duration of the disease, more severe dyspnea, greater number of involved pulmonary lobes and presence of one or more comorbid conditions. Non-influencing factors were: underweight, age, sex, smoking status and, unexpectedly, presence of Pseudomonas aeruginosa or other pathogens in sputum culture.

Conclusions: Particular attention is required for patients with bronchiectasis who have overweight, airway obstruction, longer duration of the disease, more severe dyspnea, the greater number of involved pulmonary lobes and presence of one or more comorbid conditions in order to correct modifiable risk factors of future exacerbations.
\end{abstract}

KEY WORDS: bronchiectasis, non-cystic fibrosis bronchiectasis, exacerbation, frequent exacerbator, exacerbation predictors

Wiad Lek. 2020;73(8):1717-1722

\section{INTRODUCTION}

Bronchiectasis (B) is a chronic inflammatory lung disease characterized by recurrent cough, sputum production, and recurrent airway infections [1]. Previously B considered as a rare or orphan disease, but now the prevalence has been reported up to 566 per 100000 population, moreover, over the past 10 years the incidence has been enlarged by $40 \%$ [2]. The common pathophysiological pathway of B consists of Cole's "vicious cycle" hypothesis of infection, inflammation and airway structural changes [3]. Exacerbations of B defined clinically as worsening of the usual respiratory symptoms (sputum quantity, sputum purulence, or dyspnea) requiring treatment with antibiotics $[4,5]$. They are associated with accelerated lung function decline and deterioration in quality of life [6]. Exacerbations are important events in the natural history of $\mathrm{B}$ and their management imposes a significant economic burden on the healthcare system. Patients with three or more exacerbations a year had worse health status, were more likely to be hospitalized for treatment, and had increased mortality [7]. Published data suggest that the number of exacerbations per year independently predicts future risk of mortality [8], while patients with three or more exacerbations per year have double mortality rate compared to those who do not experience exacerbations [9]. Thus, B exacerbations are both an indicator of severity and a driver of disease progression, but a great deal more data are needed to fully understand their causes and consequences [10]. In clinical practice, it is important to identify patients who are at risk of future exacerbations to plan preventative therapy appropriately [11]. International guidelines for B recommend treatment with, for example, inhaled or oral prophylactic antibiotic therapy for patients with three or more exacerbations per year to prevent worsening of the clinical symptoms $[12,13]$, but at this time point, the evidence background is quite small. To date, the relationships between the frequency of exacerbations and chronic colonization of Pseudomonas aeruginosa (PA) [14], as well as high neutrophil elastase activity [15], an elevated level of cathelicidin LL-37 and reduced Secretory Leukocyte Protease Inhibitor levels [16] are described in the frequent exacerbator phenotype. Low forced expiratory volume in the first second $\left(\mathrm{FEV}_{1}\right)$, radiological severity of disease and coexisting chronic obstructive pulmonary disease are also described as independent predictors of future exacerbation frequency [10]. Searching for other specific markers, which could predict the risk of exacerbations, is still in progress [17].

\section{THE AIM}

The study aimed to determine which other specific factors could predict the risk of $\mathrm{B}$ exacerbations. 
Table 1. Demographic and anthropometric data and outcome measures with values for patients with $B$.

\begin{tabular}{|c|c|c|c|c|}
\hline Variables & $\begin{array}{c}\text { Total } \\
(n=47)\end{array}$ & $\begin{array}{l}\text { Frequent exacerbators } \\
\qquad \geq 3(n=24)\end{array}$ & $\begin{array}{l}\text { Nonfrequent exacerbators } \\
\qquad<3(n=23)\end{array}$ & $p^{\mathrm{a}}$ \\
\hline Age, years (median (IQR)) & $56(38 ; 65)$ & $58(42.5 ; 64.5)$ & $44(36 ; 62)$ & $0.2^{c}$ \\
\hline Male sex (n (\%)) & $12(25.5)$ & $6(25)$ & $6(26.1)$ & $0.39^{\mathrm{d}}$ \\
\hline $\mathrm{BMI}, \mathrm{kg} / \mathrm{m}^{2}($ Mean $(\mathrm{SD}))$ & $24.3(4.4)$ & $25.7(4.8)$ & $22.9(3.6)$ & $0.03^{e}$ \\
\hline $\mathrm{BMI}<18.5 \mathrm{~kg} / \mathrm{m}^{2}(\mathrm{n}(\%))$ & $5(10.6)$ & $3(12.5)$ & $2(8.7)$ & $0.08^{\mathrm{d}}$ \\
\hline $\mathrm{BMI} \geq 25 \mathrm{~kg} / \mathrm{m}^{2}(\mathrm{n}(\%))$ & $21(44.7)$ & $16(66.7)$ & $5(21.7)$ & $0.01^{d}$ \\
\hline Never smoked (n (\%)) & $36(76.6)$ & $20(83.3)$ & $16(69.6)$ & $0.88^{\mathrm{d}}$ \\
\hline $\begin{array}{l}\text { Confirmed disease duration, years } \\
\text { (median (IQR)) }\end{array}$ & $2(0.2 ; 6)$ & $6(0.37 ; 12.5)$ & $0.42(0.08 ; 2)$ & $0.01^{c}$ \\
\hline $\begin{array}{l}\text { History of hospital admissions due to } \\
\text { exacerbations } 12 \text { months before study ( } \mathrm{n}(\%))\end{array}$ & $30(63.8)$ & $20(83.3)$ & $10(43.5)$ & $0.02^{\mathrm{d}}$ \\
\hline mMRC, scores (median (IQR)) & $1(1 ; 2)$ & $1(1 ; 2)$ & $1(1 ; 1)$ & $0.01^{c}$ \\
\hline \multicolumn{5}{|c|}{ Pulmonary function testing } \\
\hline $\mathrm{FEV}_{1}, \%$ predicted (mean (SD)) & $81.6(28.1)$ & $72(30.1)$ & $91.7(22.4)$ & 0.02 \\
\hline FVC, \% predicted (mean (SD)) & $104.3(22.8)$ & $100.4(22.8)$ & $108.3(22.5)$ & $0.24^{\mathrm{e}}$ \\
\hline $\mathrm{FEV}_{1} / \mathrm{FVC}$ ratio (\%) (median (IQR)) & $65.1(47.9 ; 72.5)$ & $52.5(39.2 ; 67.4)$ & $72.5(65 ; 79.4)$ & $0.0003^{c}$ \\
\hline $\mathrm{FEV}_{1} / \mathrm{FVC}$ ratio < $0.7(\mathrm{n}(\%))$ & $29(61.7)$ & $19(79.2)$ & $10(43.5)$ & 0.053 \\
\hline \multicolumn{5}{|c|}{ Airflow limitation stages in patients with $\mathrm{FEV} / \mathrm{FVC}$ ratio $<0.7$ (n (\%)) } \\
\hline Stage $1\left(\mathrm{FEV}_{1} \geq 80 \%\right.$ predicted) $(\mathrm{n}(\%))$ & $8(27.6)$ & $1(5.3)$ & $7(70)$ & $0.002^{\mathrm{d}}$ \\
\hline $\begin{array}{c}\text { Stage } 2\left(\mathrm{FEV}_{1} 50 \%-80 \% \text { predicted) }\right. \\
(\mathrm{n}(\%))\end{array}$ & $16(55.2)$ & $13(68.4)$ & $3(30)$ & $0.226^{d}$ \\
\hline $\begin{array}{c}\text { Stage } 3\left(\mathrm{FEV}_{1}, 30 \%-50 \% \text { predicted) }\right. \\
(\mathrm{n}(\%))\end{array}$ & $4(13.8)$ & $4(21)$ & $0(0)$ & $0.638^{d}$ \\
\hline Stage $4\left(\mathrm{FEV}_{1}<30 \%\right.$ predicted $)(\mathrm{n}(\%))$ & $1(3.4)$ & $1(5.3)$ & $0(0)$ & $0.52^{\mathrm{d}}$ \\
\hline $\mathrm{SpO}_{2}(\%)($ median $(\mathrm{IQR}))$ & $96(94 ; 97)$ & $95(94 ; 96)$ & $96(96 ; 97)$ & $0.01^{c}$ \\
\hline Quantity of involved lobes (median (IQR)) & $2(2 ; 3)$ & $3(2 ; 3.5)$ & $2(1 ; 3)$ & $0.054^{c}$ \\
\hline Pseudomona's colonization (n (\%)) & $11(23.4)$ & $7(29.2)$ & $4(17.4)$ & $0.914^{d}$ \\
\hline Colonisation with other organisms (n (\%)) & $20(42.6)$ & $10(41.7)$ & $10(43.5)$ & $0.269^{d}$ \\
\hline \multicolumn{5}{|c|}{ The prevalence of comorbidity } \\
\hline Presence of any comorbid condition $\mathrm{n}(\%)$ & $43(91.5)$ & $24(100)$ & $19(82.6)$ & $0.21^{\mathrm{d}}$ \\
\hline $\begin{array}{l}\text { Presence of respiratory comorbid } \\
\text { condition } \mathrm{n}(\%)\end{array}$ & $35(74.5)$ & $21(87.5)$ & $14(60.8)$ & $0.16^{\mathrm{d}}$ \\
\hline $\begin{array}{l}\text { Presence of more than one comorbid } \\
\text { condition } \mathrm{n}(\%)\end{array}$ & $30(63.8)$ & $19(79.2)$ & $11(47.8)$ & $0.11^{d}$ \\
\hline \multicolumn{5}{|c|}{ BSI stages $(\mathrm{n}(\%))$} \\
\hline Mild (0-4 scores) & $10(21.3)$ & $0(0)$ & $10(43.5)$ & $0.002^{\mathrm{d}}$ \\
\hline Moderate (5-8 scores) & $13(27.7)$ & $4(16.7)$ & $9(39.1)$ & $0.326^{d}$ \\
\hline Severe ( $\geq 9$ scores) & $24(51)$ & $20(83.3)$ & $4(17.4)$ & $0.00005^{d}$ \\
\hline \multicolumn{5}{|c|}{ FACED stages $(\mathrm{n}(\%))$} \\
\hline Mild (0-2 scores) & $29(61.7)$ & $12(50)$ & $17(73.9)$ & $0.335^{d}$ \\
\hline Moderate (3-4 scores) & $14(29.8)$ & $8(33.3)$ & $6(26.1)$ & $0.354^{d}$ \\
\hline Severe (5-7 scores) & $4(8.5)$ & $4(16.7)$ & $0(0)$ & $0.254^{d}$ \\
\hline
\end{tabular}

a comparing frequent exacerbators and non-frequent exacerbators;

'Mann Whitney U test;

${ }^{d}$ Chi-squared test; et-test; BMI: body mass index; SD: standard deviation; frequent exacerbators include $\geq 3$ exacerbations per year, and non-frequent exacerbators include < 3 exacerbations per year; mMRC: Modified Medical Research Council Dyspnea Scale; BSI (Bronchiectasis Severity Index) and FACED (FEV1, age, PA colonization, radiological extension and dyspnea) scales for bronchiectasis severity assessment. 


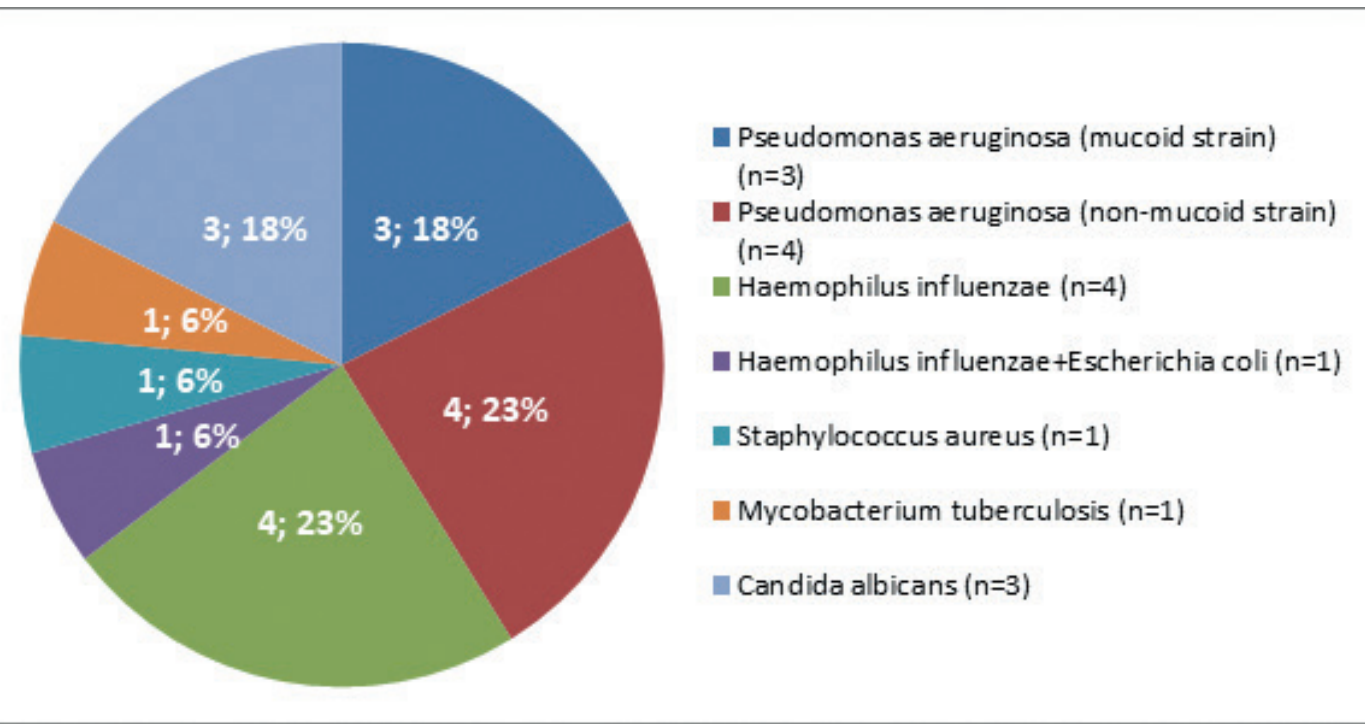

Fig. 1. Detected pathogens in the sputum of Frequent exacerbators (group 1).

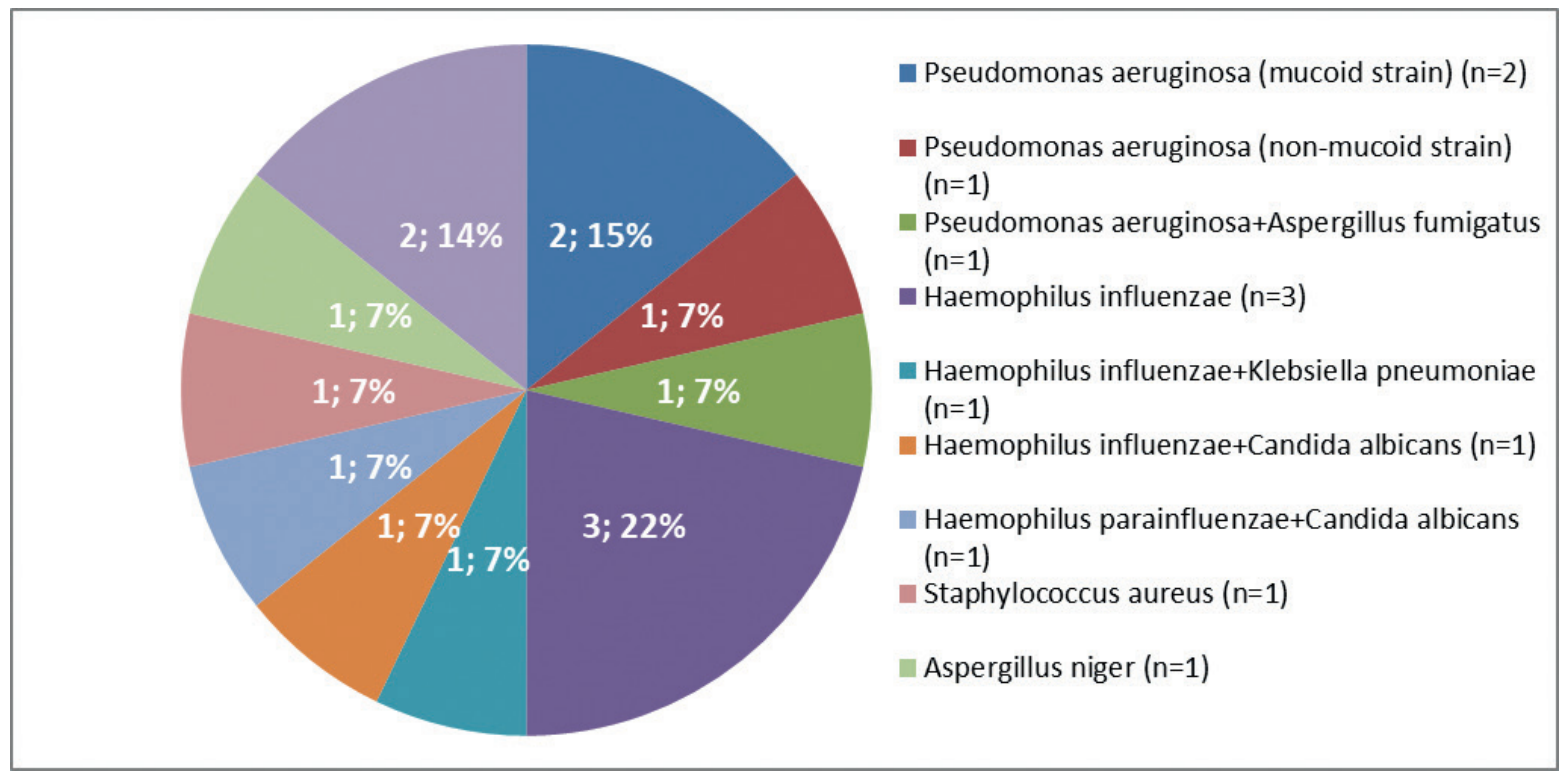

Fig. 2. Detected pathogens in the sputum of non-Frequent exacerbators (group 2).

\section{MATERIALS AND METHODS}

\section{STUDY POPULATION}

Patients with $B$ were prospectively recruited into the study in Dnipro region in Department of Occupational Diseases and Clinical Immunology of State Establishment "Dnipropetrovsk medical academy of the Ministry of Health of Ukraine" from October 2018 to July 2019. Inclusion criteria: adult males and females with B confirmed by high resolution computed tomography (HRCT) based on the following criteria: 1) lack of tapering within the bronchi; 2) dilation of the bronchi wherever the inner diameter was larger than that of the adjacent pulmonary artery, or 3) visualisation of the peripheral bronchi at intervals one centimeter of the costal pleural surface or the adjacent mediastinal pleural surface [18]. Stable phase of B (no change in symptoms and medication for at least 8 weeks) was required. Exclusion criteria: cystic fibrosis, active tuberculosis, history of any malignancy, pregnancy and lactation, history of lung transplantation.

\section{STUDY GROUP}

47 patients (caucasians) were enrolled in the study. Most of them were women $(p=0.003)$, mostly non-smokers $(p=0.001)$, more than half had signs of airway obstruction $(p=0.024)$. Depending on the number of exacerbations per previous year, patients were divided into two groups: frequent exacerbators - $\geq 3$ cases per year $(\mathrm{n}=24)$ and non-frequent exacerbators $-<3$ cases per year $(n=23)$.

\section{METHODS}

Demographic and anthropometric data of all patients were registered: gender, age, height by the stadiometer RP-2000, weight using the scales "Body composition monitor Omron 
BF511" for the static weighing. Body mass index (BMI) was calculated by the conventional formula [19]. Medical history (duration of the disease from the moment the diagnosis is confirmed, frequency of exacerbations during the previous year and hospital admissions during the previous 12 months, presence of comorbidity) was detected in medical source documentation. Smoking status was self-reported. Dyspnea was assessed using the Modified Medical Research Council Dyspnea Scale [20].

Sputum samples were accepted if they contained $<10$ squamous epithelial cells per low-powered field. Microbiological detection of sputum samples was conducted by conventional bacteriological methods [21]. In most cases we examined spontaneous sputum $(n=40)$, in cases where the patients did not produce sputum, we examined induced sputum $(n=7)$.

For all patients baseline, the respiratory function was measured by computed spirometry using the MasterLab (Viasis, Germany). Post-bronchodilation tests were evaluated in 15 minutes after administration of $400 \mu \mathrm{g}$ of salbutamol by pressurized metered-dose inhaler via a spacer. Predicted and absolute values of $\mathrm{FEV}_{\text {, }}$, forced vital capacity (FVC) and $\mathrm{FEV}_{1} / \mathrm{FVC}$ ratio were calculated by ATS and ERS 2005 standards. Blood oxygen saturation $\left(\mathrm{SpO}_{2}\right)$ was measured in sitting patients after a 30-minute rest by puls oximeter Bitmos Sat 816 MasimoSET.

The severity of the disease was determined using Bronchiectasis Severity Index (BSI), which consisted of age, BMI, HRCT score (independent predictor of future hospitalization was three or more lobes involving), $\mathrm{FEV}_{1}$, Medical Research Council dyspnoea score, bacterial colonisation ( $P A$ or other pathogenic bacteria), prior hospital admission and exacerbations ( 3 or more exacerbations are suggested as independent predictor of mortality and future hospitalizations) [22] and using FACED score, which comprises $\mathrm{FEV}_{1}(\mathrm{~F})$, age (A), PA colonization (C), radiological extension (E) (two or more lobes involving suggested as significant value) and dyspnea (D) [23].

\section{STATISTICS}

Statistical analyses were performed using the "STATISTICA 6.1» (StatSoft Inc., USA, № AGAR909 E415822FA). The data were presented as mean (standard deviation, SD) and median (interquartile range, IQR) in the case of quantitative variables, or as absolute numbers and percentages $(\mathrm{n}, \%)$ in the case of qualitative variables. The distribution of variables was analysed using the Shapiro-Wilk test. The Chi-squared test was used to test the differences in discrete variables. Whereas for continuous variables, the t-test or Mann-Whitney U test was used. Spearman's coefficient was calculated to assess the correlation between variables. The $95 \%$ confidence interval was calculated for the independent variables, with $\mathrm{p} \leq 0.05$ considered to be significant.

Authors declare that the research was conducted according to the principles of the World Medical Association Declaration of Helsinki "Ethical Principles for Medical Research Involving Human Subjects" (amended in Oc- tober 2013). Approvals were obtained from the ethics committee of the State Establishment "Dnipropetrovsk medical academy of the Ministry of Health of Ukraine". Written informed consent was obtained from all patients who participated in this study.

\section{RESULTS AND DISCUSSION}

Demographic, anthropometric and clinical characteristics of the patients are presented in table 1.

Both groups were similar by age, sex and smoking status, no correlation was found between the number of exacerbations and age $(\mathrm{R}=0.1, p=0.48)$, $\operatorname{sex}(\mathrm{R}=0.02, p=0.88)$, smoking status $(\mathrm{R}=-0.19, p=0.19)$.

Patients with increased BMI prevailed in the total sample. The average BMI was statistically significantly higher in the group of frequent exacerbators, in addition, the proportions of overweight patients were also significantly higher in the group of frequent exacerbators and there was a direct correlation between the number of exacerbations and BMI $(\mathrm{R}=0.39, p=0.006)$. The duration of the disease was statistically different too - frequent exacerbators were sick much longer at the time of the study, also direct correlation between disease duration and exacerbation frequency was detected $(\mathrm{R}=0.43, p=0.003)$. More than half of the patients had a history of hospitalization for $\mathrm{B}$, however, there was a significantly higher hospitalization rate among frequent exacerbators. Non-frequent exacerbators assessed their dyspnea by the mMRC less severe than frequent exacerbators, the correlation between shortness of breath by the $\mathrm{MMRC}$ and exacerbation number was direct $(\mathrm{R}=0.48, p=0.003)$.

Almost all patients in both groups had normal FVC, however, frequent exacerbators more often had signs of airways obstruction, including significantly lower values of $\mathrm{FEV}_{1}$ and $\mathrm{FEV}_{1} / \mathrm{FVC}$ ratio. Analysis of $\mathrm{FEV}_{1}$ values in patients with reduced $\mathrm{FEV}_{1} / \mathrm{FVC}$ ratio revealed that among non-frequent exacerbators the first stage of obstruction prevails, unlike frequent exacerbators among whom $72.4 \%$ of patients have obstruction of the second stage and higher. There was found negative correlation between exacerbation frequency and $\mathrm{FEV}_{1} \mathrm{R}=-0.39, p=0.005$, and $\mathrm{FEV}_{1} / \mathrm{FVC}$ ratio, $\mathrm{R}=-0.47, p=0.0008$ ).

$\mathrm{SpO}_{2}$ was normal in almost all patients, but the comparison of the values showed that among the frequent exacerbators $\mathrm{SpO}_{2}$ was statistically lower.

The number of involved lung lobes is one of the main criteria for calculating the severity of B according to both BSI and FACED scales. The patients in both groups on average had two or more affected lobes and the difference in the number of involved lobes between two groups was not statistically significant. However, the correlation between exacerbation frequency and number of involved lobes was identified $(\mathrm{R}=0.37, p=0.013)$.

As for sputum microbiota, pathogens were detected in 31 (66\%) samples. The most common pathogens were Pseudomonas aeruginosa and Haemophilus influenzae in both groups. The proportions of detected pathogens in groups 
are presented in figures 1 and 2. The difference between the proportions of patients with Pseudomonas aeruginosa and other pathogens in sputum in both groups was not statistically significant. Also no statistically significant correlation was found between the presence of $P A(\mathrm{R}=0.16$, $p=0.27)$ in sputum or any other pathogen $(\mathrm{R}=-0.078$, $p=0.59)$ and the number of exacerbations.

Comorbidity was detected in $43(91.5 \%)$ patients, 30 of them $(63.8 \%)$ had more than one comorbid condition. 35 patients with comorbidity had respiratory pathology $(81.4 \%)$, among them chronic obstructive pulmonary disease (COPD) is in 24 patients (55.8\%), asthma - in nine patients $(20.9 \%)$, rhinosinusitis - in 14 patients $(32.6 \%)$, tracheobronchomegaly - in one patient $(2.3 \%)$, primary ciliary dyskinesia - in two patients (4.6\%), atrophic bronchitis in one patient (2.3\%).

Among the cardiovascular diseases prevails arterial hypertension - in 10 patients $(23.3 \%)$, there is also heart arrhythmia in two patients (4.6\%), chronic heart failure in one patient $(2.3 \%)$, chronic rheumatic heart disease in one patient $(2.3 \%)$ and stable ischemic heart disease in one patient $(2.3 \%)$. Among diseases of other organs and systems prevailed gastro-oesophageal reflux disease - in three patients $(6.9 \%)$, chronic pancreatitis in two patients (4.6\%), type 2 diabetes in two patients (4.6\%). Also benign prostatic hyperplasia was present in one patient $(2.3 \%)$, thrombocytopenia - in one patient $(2.3 \%)$, secondary erythrocytosis in one patient (2.3\%), glomerulonephritis in one patient $(2.3 \%)$, atrophic gastritis in one patient $(2.3 \%)$ and thyroid cyst in one patient $(2.3 \%)$. According to the data presented in table 1 , there was not detected statistically significant difference between the frequencies of presence of one or more than one comorbid conditions in groups of frequent exacerbators and non-frequent exacerbators, however, the correlation was found between the number of exacerbations and presence of any comorbidity condition $(\mathrm{R}=0.39, p=0.007)$, presence of respiratory comorbidity condition $(\mathrm{R}=0.44, p=0.002)$ and presence of more than one comorbidity condition $(\mathrm{R}=0.41, p=0.004)$.

Among the frequent exacerbators, there were statistically significantly more severe patients and less mild patients according to the BSI scale. At the same time, no differences between the two groups in patients' severity were found by FACED scale. But, there was detected a moderate correlation between the exacerbation frequency and severity of the disease assessed by BSI $(\mathrm{R}=0.68, p=0.0000001)$ and weak correlation between the exacerbation frequency and severity of the disease assessed by FACED scale $(\mathrm{R}=0.36, p=0.01)$.

\section{CONCLUSIONS}

The population of patients with B in Dnipro region is mainly people of working age, more often women. We found out that such factors as age, sex and history of smoking do not affect the frequency of exacerbations in these patients. Unexpectedly our study showed, that the presence of pathogens in sputum, in particular, $P A$ also is not a statistically significant predictor of more frequent exacerbations. Perhaps this may be due to the fact that such patients are more likely to suffer from a productive cough with purulent sputum, due to which, in turn, it is difficult for them to determine the exacerbation. While patients without $P A$ colonization are easier to independently determine the exacerbation and seek medical help.

At the same time, we discovered that overweight even more than underweight can be an independent predictor of more frequent exacerbations. In our study frequent exacerbators had a longer duration of the disease from the moment the diagnosis was confirmed, more severe dyspnea and airways obstruction, lower blood oxygen saturation and more severe disease by BSI than non-frequent exacerbators. Furthermore, the number of exacerbations increased with the number of affected lung lobes and correlated with the presence of respiratory or non-respiratory comorbidity. So exactly these factors could be proposed to consider as predictors of frequent exacerbations in adult patients with bronchiectasis.

\section{REFERENCES}

1. Chalmers JD, AlibertiS, Blasi F. Management of bronchiectasis in adults. EurRespir J 2015;45:1446-1462.

2. Quint JK, Millett ER, Joshi M, et al. Changes in the incidence, prevalence and mortality of bronchiectasis in the UK from 2004 to 2013: a populationbased cohort study. EurRespir J 2016;47:186-93.

3. P.J.Cole, Inflammation:a two-edged sword-the model of bronchiectasis, Eur. J. Respir. Dis 1986:147,6-15.

4. Hill AT, Haworth CS, Aliberti $S$, et al. Pulmonary exacerbation in adults with bronchiectasis: a consensus definition for clinical research. EurRespir J 2017;49:1700051.

5. Wong C, Jayaram L, Karalus N, et al. Azithromycin for prevention of exacerbations in non-cystic fibrosis bronchiectasis (EMBRACE): a randomised, double-blind, placebo-controlled trial. The Lancet 2012;380(9842), 660-667. doi:10.1016/s0140-6736(12)60953-2.

6. Redondo M, FerriS, Chalmers J. Exacerbations of bronchiectasis in adults. Community Acquir. Infect 2016;3(2),43-50.

7. Chalmers JD, Aliberti S, Filonenko A, et al. Characterization of the "Frequent Exacerbator Phenotype" in bronchiectasis. Am J Respir Crit Care Med 2018;197:1410-20.

8. Pertseva T., Gashynova K., Dmytrychenko V. et al. Bronchoectatic disease: the state of art and the clinical case. Medical perspectives 2018;23(3):153-161.

9. Chalmers JD, Goeminne P, Aliberti S, et al. The bronchiectasis severity index: an international derivation and validation study. Am J Respircrit Care Med 2014;189:576-585.

10. James D. Chalmers. Bronchiectasis Exacerbations Are HeartBreaking. Ann Am Thorac Soc 2018;15(3),301-303. D0l: 10.1513/ AnnalsATS.201710-832ED.

11. Chalmers JD, Aliberti S, Filonenko A, et al. Characterisation of the "frequent exacerbator phenotype" in bronchiectasis. American Journal of Respiratory and Critical Care Medicine 2017:197(11),1410-1420. doi:10.1164/rccm.201711-22020c

12. Polverino E, Goeminne $P C, M C D$ onnell et al. European Respiratory Society guidelines for the management of adult bronchiectasis. EurRespir J. 2017;50(3):1700629. doi: 10.1183/13993003.00629-2017.

13. Pasteur MC, Bilton D, Hill AT, British Thoracic Society Bronchiectasis non-CF Guideline Group. British Thoracic Society guideline for non-CF bronchiectasis. Thorax 2010; 65(1): i1-58. 
14. Stone GA, Blanchette CM, Patel R, et al. Pseudomonas Aeruginosa Is Associated with Greater Frequency of Exacerbations Among Patients with Non-Cystic Fibrosis Bronchiectasis in the United States. American Journal of Respiratory and Critical Care Medicine 2019;199:A5706.

15. Shoemark A, Cant E, Carreto L, et al. A point of care neutrophil elastase activity assay identifies bronchiectasis severity, airway infection and risk of exacerbation. Eur Respir J 2019;53:1900303. doi:10.1183/13993003.00303-2019.

16. Sibila 0, Perea L, Cantó E, et al. Antimicrobial peptides, disease severity and exacerbations in bronchiectasis. Thorax 2019;74:835-842. doi:10.1136/thoraxjnl-2018-212895.

17. Gashynova K, Sanina N. COPD: Plasma surfactant protein-D (SPD) concentration as a predictor of acute exacerbations (AE) probability. European Respiratory Journal 2016;48:PA652. D0I: 10.1183/13993003. congress-2016.PA652.

18. Naidich DP, McCauley DI, Khouri NF, et al. Computed tomography of bronchiectasis. J Comput Assist Tomogr 1982;6:437-444.

19. Physical status: the use and interpretation of anthropometry. Report of aWHO Expert Committee [Text] / World Health Organization. - Geneva: World Health Organization, 1995. - $36 \mathrm{p}$.

20. Global Initiative for Chronic Obstructive Lung Diseases (GOLD). Global strategy for diagnosis, management, and prevention of chronic obstructive pulmonary disease [Electronic Resource] / Revised, 2011. - Available at : http://www.goldcopd.org/uploads/users/files/ GOLD_Report_2011_Feb21.pdf

21. Clinical and Laboratory Standards Institute (CLSI). Performance standards for antimicrobial susceptibility testing: 14th informational supplement [CLSI document M100-S14]. Wayne, Clinical and Laboratory Standards Institute, 2004.

22. Chalmers JD, Goeminne P, Aliberti S, et al. The bronchiectasis severity index. An international derivation and validation study. Am J Respir Crit Care Med 2014;189:576-585.

23. Martinez-Garcia MA, de Gracia J, Vendrell Relat M et al. Multidimensional approach to non-cystic fibrosis bronchiectasis; the FACED score. Eur Respir J 2014;43:1357-1367.

\section{ORCID and contributionship:}

Kateryna Gashynova: 0000-0003-2955-9687 A,E,F

Kseniia Suska: 0000-0001-9735-4988 A,B,C,D,F

Valeriia Dmytrychenko: 0000-0003-4337-1429 B,E,F

\section{Conflict of interest:}

The Authors declare no conflict of interest.

\section{CORRESPONDING AUTHOR}

\section{Kseniia Suska}

Occupational Diseases and Clinical Immunology,

SI "Dnipropetrovsk medical academy of the Health Ministry of Ukraine"

Dnipro, Ukraine

tel: +380951230362

e-mail: kseniiasuska@gmail.com

Received: 15.04 .2020

Accepted: 10.07 .2020

A - Work concept and design, B - Data collection and analysis, C - Responsibility for statistical analysis,

$\mathbf{D}$-Writing the article, $\mathbf{E}$ - Critical review, $\mathbf{F}$ - Final approval of the article 